\title{
Imidazoacridinone-dependent lysosomal photodestruction: a pharmacological Trojan horse approach to eradicate multidrug-resistant cancers
}

\author{
Y Adar ${ }^{1}$, M Stark ${ }^{1}$, EE Bram ${ }^{1,6}$, P Nowak-Sliwinska ${ }^{2,3}$, H van den Bergh ${ }^{2}$, G Szewczyk ${ }^{4}$, T Sarna ${ }^{4}$, A Skladanowski ${ }^{5}$, AW Griffioen ${ }^{3}$ \\ and YG Assaraf*,1
}

Multidrug resistance (MDR) remains a primary hindrance to curative cancer therapy. Thus, introduction of novel strategies to overcome MDR is of paramount therapeutic significance. Sequestration of chemotherapeutics in lysosomes is an established mechanism of drug resistance. Here, we show that MDR cells display a marked increase in lysosome number. We further demonstrate that imidazoacridinones (IAs), which are cytotoxic fluorochromes, undergo a dramatic compartmentalization in lysosomes because of their hydrophobic weak base nature. We hence developed a novel photoactivation-based pharmacological Trojan horse approach to target and eradicate MDR cancer cells based on photo-rupture of IA-loaded lysosomes and tumor cell lysis via formation of reactive oxygen species. Illumination of IA-loaded cells resulted in lysosomal photodestruction and restoration of parental cell drug sensitivity. Lysosomal photodestruction of MDR cells overexpressing the key MDR efflux transporters $A B C G 2, A B C B 1$ or $A B C C 1$ resulted in 10- to 52 -fold lower $I_{50}$ values of various IAs, thereby restoring parental cell sensitivity. Finally, in vivo application of this photodynamic therapy strategy after i.v. injection of IAs in human ovarian tumor xenografts in the chorioallantoic membrane model revealed selective destruction of tumors and their associated vasculature. These findings identify lysosomal sequestration of IAs as an Achilles heel of MDR cells that can be harnessed to eradicate MDR tumor cells via lysosomal photodestruction.

Cell Death and Disease (2012) 3, e293; doi:10.1038/cddis.2012.30; published online 5 April 2012

Subject Category: Cancer

Imidazoacridinones (IAs; Supplementary Figure 1) are hydrophobic weak base cytotoxic fluorochromes that display considerable antitumor activity towards murine and human malignant cell lines in vitro, $^{1}$ and against breast- and colon adenocarcinomas in vivo. ${ }^{2}$ The marked similarity in the chemical structure between IAs and the established topoisomerase II inhibitor mitoxantrone, suggested a common cellular drug target. Indeed, previous studies have shown that IAs block topoisomerase II activity ${ }^{1}$ and stimulated the in vitro formation of topoisomerase II-cleavable complexes. Moreover, previous studies have shown that IAs, which harbor a planar IA core, are capable of DNA intercalation. ${ }^{3}$

The frequent emergence of multidrug resistance (MDR) to structurally and functionally unrelated anticancer drugs, is a major impediment to curative cancer chemotherapy. ${ }^{4-9}$ ATP-driven MDR efflux transporters belong to the large ATP-binding cassette $(A B C)$ superfamily of transporters that include ABCB1 (P-gp), ABCC1 (MRP1) and ABCG2 (BCRP). Overexpression of these efflux pumps results in the expulsion of a multitude of chemotherapeutic drugs, thereby leading to acquisition of a broad-spectrum drug resistance phenotype known as MDR. As MDR remains a major obstacle to successful cancer chemotherapy, there is a burning need to develop new strategies to overcome MDR phenomena. Taking advantage of the increased number of lysosomes in MDR cells, we here developed an IAs-based photoactivated pharmacological Trojan horse approach to eliminate MDR cancer cells in vitro and in vivo. This strategy is based on the efficient sequestration of IAs in lysosomes. Upon photoactivation, reactive oxygen species (ROS) rapidly form, resulting in lysosomal rupture and tumor cell lysis. In vivo photodynamic therapy (PDT) experiments, based on the selective photodestruction of targeted tissue ${ }^{10}$ further established that this strategy is endowed with a potent capability to destroy human tumor xenografts and their associated vasculature.

\footnotetext{
${ }^{1}$ Department of Biology, The Fred Wyszkowski Cancer Research Laboratory, Technion-Israel Institute of Technology, Haifa, Israel; ${ }^{2}$ Institute of Chemical Sciences and Engineering, Swiss Federal Institute of Technology (EPFL), Lausanne, Switzerland; ${ }^{3}$ Angiogenesis Laboratory, Department of Medical Oncology, VU University Medical Center, Amsterdam, The Netherlands; ${ }^{4}$ Department of Biophysics, Jagiellonian University, Krakow, Poland and ${ }^{5}$ Department of Pharmaceutical Technology and Biochemistry, Gdansk University of Technology, Gdansk, Poland

*Corresponding author: YG Assaraf, Department of Biology, The Fred Wyszkowski Cancer Research Laboratory, Technion-Israel Institute of Technology, Haifa 32000, Israel. Tel: + 9724 8293744; Fax: + 9724 8225153; E-mail: assaraf@tx.technion.ac.il

${ }^{6}$ Current address: Weiss Lab for Synthetic Biology, Department of Biological Engineering, Massachusetts Institute of Technology, Cambridge, MA, USA.

Keywords: cancer chemotherapy; multidrug resistance; hydrophobic weak base drugs; lysosomal sequestration; photodynamic therapy; reactive oxygen species Abbreviations: ABC, ATP-binding cassette; IA, imidazoacridinone; FTC, fumitremorgin C; MDR, multidrug resistance; PDT, photodynamic therapy; ROS, reactive oxygen species

Received 17.1.12; revised 21.2.12; accepted 21.2.12; Edited by A Stephanou
} 


\section{Results}

IAs specifically accumulate in lysosomes. Recently we have shown that IAs (Supplementary Figure 1), including C-1330, C-1375 and C-1379, are not recognized by ABCG2, whereas their hydroxyl-containing homologs (e.g., C-1311) are readily expelled by this multidrug efflux pump. ${ }^{11}$ Hence, on the basis of their hydrophobic weak base nature and their structural similarity to acridine orange, an established fluorophore known to concentrate within lysosomes, we hypothesized that these IAs may also accumulate within acidic organelles such as lysosomes. We therefore exposed parental A549 cells and their ABCG2-overexpressing MDR subline A549/K1.5 ${ }^{11}$ to $\mathrm{C}-1330$ (green fluorescence) and LysoTracker red (red fluorescence), an established viable lysosomal marker. Cells were counterstained with the supravital dye Hoechst 33342 (blue fluorescence). Both cells displayed co-localization of the red and green fluorescence, resulting in an orange signal in the merged photographs (Figures 1a-f). Identical results were obtained with the IAs C-1375 and C-1379 (data not shown). Moreover, we observed a sevenfold increase in lysosomes' fluorescence (i.e., an increase in the number and volume of lysosomes) in MDR A549/K1.5 cells $(4500 \pm 937$ a.u./cell), relative to parental A549 cells $(600 \pm 317$ a.u./cell) as determined by LysoTracker red staining and quantification using the EZ-Quant software (EZ-Quant, Tel-Aviv, Israel) (Figure 1g). Viable staining of the mitochondrial marker MitoTracker red (red fluorescence) in A549 cells excluded the possibility of C-1330 localization in mitochondria (Supplementary Figure 2).

We postulated that the acidic $\mathrm{pH}$ of lysosomes is the driving force for the marked compartmentalization of IAs in lysosomes. We hence used two independent approaches to alkalinize lysosomes: ammonium chloride, a weak base lysosomotropic alkalinization agent and bafilomycin A1, a potent inhibitor of $\mathrm{H}^{+}$-ATPase (i.e., vesicular ATPase). Following preincubation of A549 (Figures 2a, c and e) and A549/K1.5 cells (Figures 2b, d and f) with ammonium chloride (Figures $2 \mathrm{c}$ and $\mathrm{d}$ ) or bafilomycin A1 (Figures $2 e$ and f), subsequent exposure to $\mathrm{C}-1330$ did not result in lysosomal accumulation of $\mathrm{C}-1330$. Interestingly, under these conditions, C-1330, which bears a planar acridone ring structure that is typical of various DNA dyes, brightly stained the nuclei of both A549 and A549/K1.5 cells, in agreement with previous studies. $^{3,11}$

Photosensitization of $\mathrm{C}$-1330-treated cells results in rapid lysosomal destruction mediated by ROS. Switching on the light of the fluorescence microscope resulted in a rapid destruction and disappearance of fluorescent lysosomes in C-1330-treated cells (Supplementary Movies 1 and 2). These lysosomal bursts were visible as multiple asynchronous flashes in both A549 and A549/K1.5 cells and resulted in the disappearance of all fluorescent lysosomes over a period of a few minutes at the applied conditions. The disappearance of lysosomes was accompanied by a simultaneous gradual increase in nuclear fluorescence. We hypothesized that the rapid rupture of lysosomes and consequent release of $\mathrm{C}-1330$ was due to an intrinsic photosensitizing activity of C-1330. Photosensitizers have their destructive activity often through the formation of ROS, which may be responsible for lysosomal
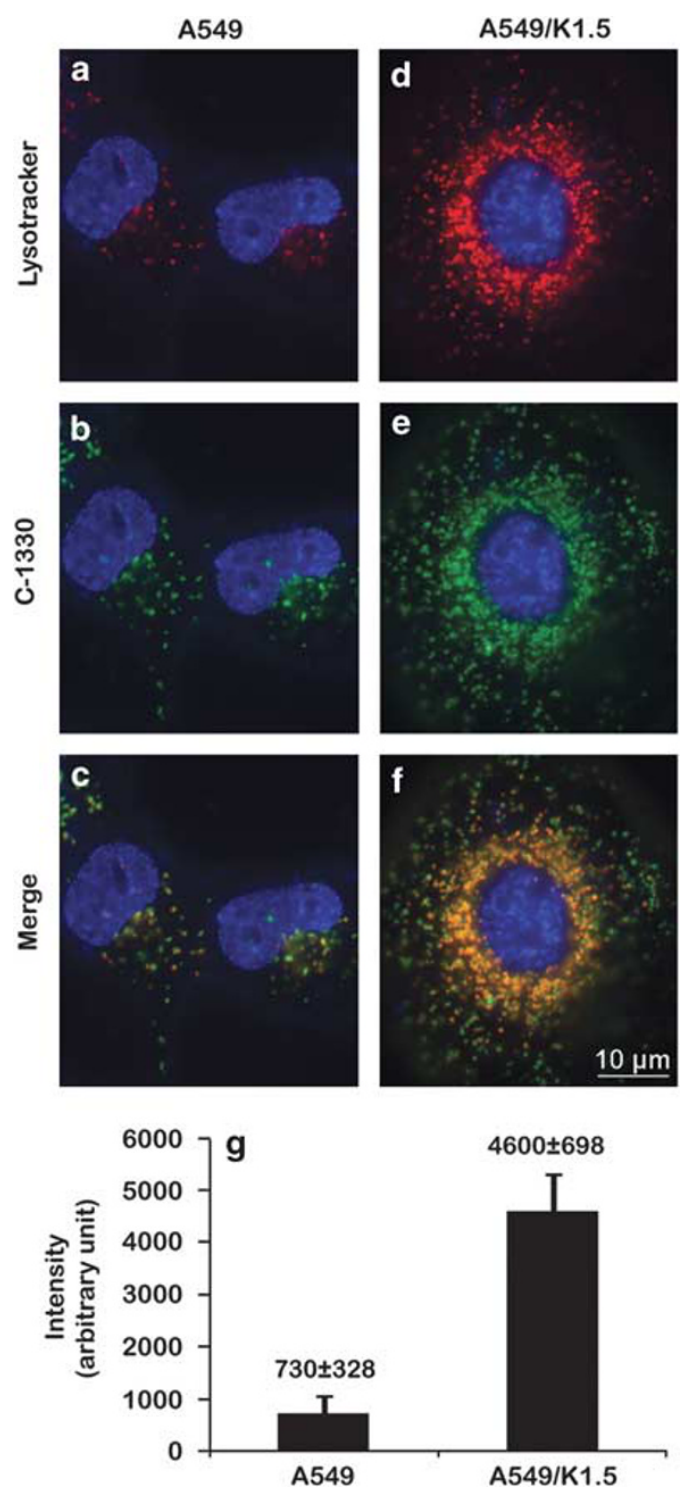

Figure 1 Co-localization of C-1330 and LysoTracker red in lysosomes in A549 and A549/K1.5 cells. Parental A549 (a-c) and their MDR subline A549/K1.5 (d-f) were viably stained with Hoechst 33342 (blue nuclear fluorescence) along with either $100 \mathrm{nM}$ LysoTracker red for $1 \mathrm{~h}$ (red fluorescence; $\mathbf{a}$, and d) or $10 \mu \mathrm{M} \mathrm{C}-1330$ (green fluorescence; $\mathbf{b}$, and $\mathbf{e}$ ) for $30 \mathrm{~min}$. Viable cell staining and quantification of the number of red fluorescent lysosomes per cell were carried out by first staining with $100 \mathrm{nM}$ LysoTracker red for $1 \mathrm{~h}(\mathbf{g})$ followed by lysosome counting in at least 25 cells from each of three independent experiments using the EZ-Quant software. Inverted fluorescence microscopy analysis of viable cell staining was performed at a magnification of $\times 630$

membrane lysis. Using direct detection of the luminescence centered at $1270 \mathrm{~nm}$, we measured the generation of singlet oxygen using C-1375 and C-1379 after pulsed excitation with $355 \mathrm{~nm}$ laser radiation in acetonitrile and DMSO (Supplementary Figure 3A). Measurable luminescence with lifetime of the order of $3 \mu$ s was only detected in samples that were saturated with either oxygen or air. The lifetime of the luminescence was consistent with the reported lifetime of singlet oxygen in this solvent. ${ }^{12}$ Moreover, using electron paramagnetic resonance (EPR)-spin trapping and 5,5-dimethyl-1-pyrroline $\mathrm{N}$-oxide (DMPO) as a spin trap, we observed photogeneration of superoxide anion by both 

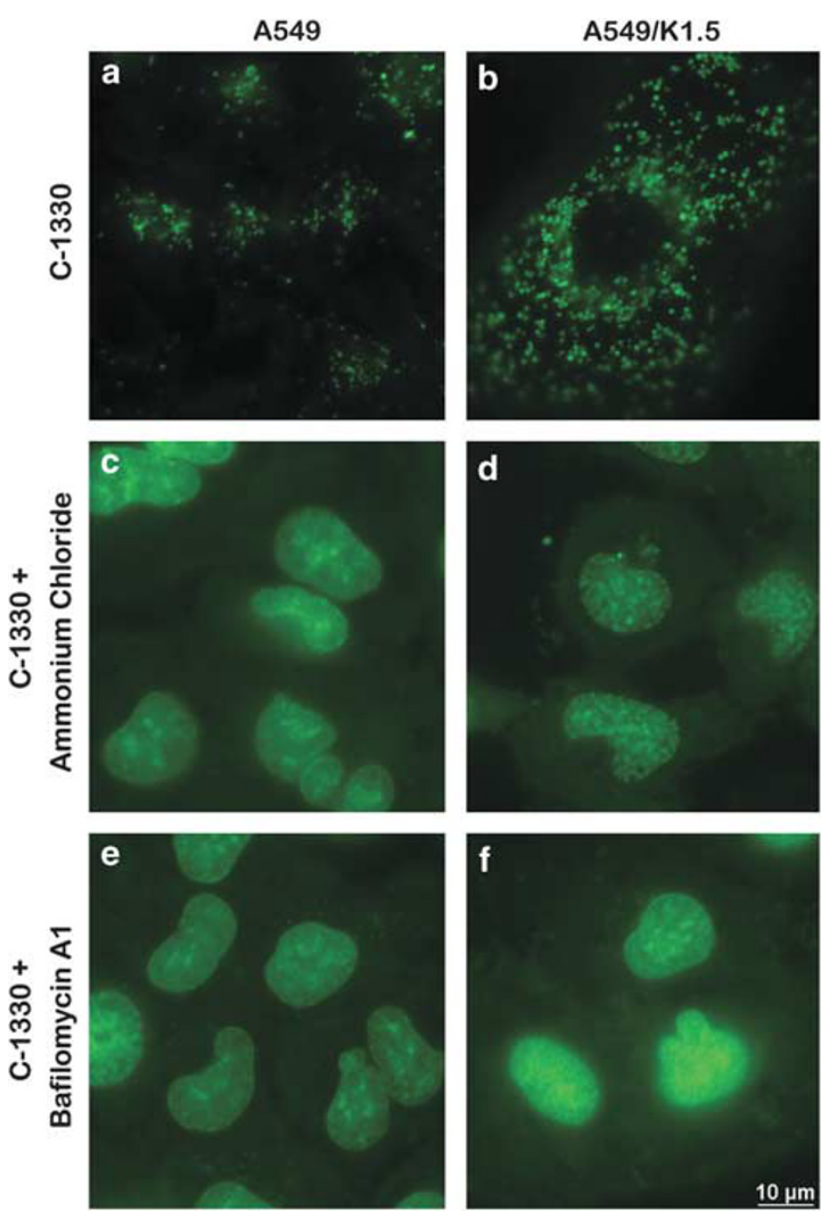

Figure 2 Lysosomotropic alkalinization agents abolish intralysosomal accumulation of C-1330. A549 (a, c and e) and A549/K1.5 cells (b, d and f) were preincubated either with the lysosomotropic agent ammonium chloride $(10 \mathrm{mM})$ for $30 \mathrm{~min}$ (c and $\mathbf{d}$ ), with bafilomycin $\mathrm{A} 1(1 \mu \mathrm{M})$ for $1 \mathrm{~h}$ (e and $\mathbf{f})$ or with none (a and $\mathbf{b}$ ), following which cells were further incubated for 30 additional min with C-1330 $(10 \mu \mathrm{M})$. Cells were then analyzed by fluorescence microscopy at a magnification of $\times 630$

C-1375 and $\mathrm{C}-1379$ in a mixture of DMSO and $\mathrm{H}_{2} \mathrm{O}(9: 1)$; Supplementary Figure 3B shows the EPR spectra of the DMPO spin adduct that evolved with irradiation of the samples in the resonant cavity. The EPR signal of the DMPO spin adduct became much stronger in the presence of $1 \mathrm{mM} \mathrm{NADH}$. Simulation of the EPR signal using magnetic parameters commonly used for the DMPO-OOH adduct, is fully consistent with the generation and spin trapping of superoxide anion. The accumulation of DMPO-OOH with irradiation time for C-1375 and $\mathrm{C}-1379$ in the absence or presence of NADH is shown in Supplementary Figures $3 \mathrm{~A}$ and $\mathrm{B}$ respectively. Whereas, the accumulation of DMPO-OOH with irradiation time for riboflavin was determined in the presence of EDTA (Supplementary Figures 3B). From such data one can calculate relative rates of the spin adduct formation in samples of the studied compounds in comparison with riboflavin/EDTA, which is a known generator of superoxide anion. It is evident from these results that these IAs can generate singlet oxygen and superoxide anion upon photoexcitation, particularly in the presence of an electron donor such as NADH. Due to the ability of superoxide anion to dismutate, it can be postulated that hydrogen peroxide will also be generated after photoexcitation of IAs. These results suggest that IAs-dependent lysosomal photodestruction is mediated by the generation of ROS, which presumably leads to the rapid rupture of the lysosomal membrane.

Photosensitization markedly enhances the cytotoxic effect of IAs and overcomes MDR. We next explored the impact of photosensitization of C-1330-loaded lysosomes on cell viability. Monolayers of A549 and A549/K1.5 cells (Figures $3 a$ and $b$, respectively) were first incubated with C-1330, washed with drug-free medium, subjected to light from the fluorescence microscope and further incubated for $48 \mathrm{~h}$. Light microscopy of Crystal Violet-stained cells revealed that cells in light-exposed areas were completely lysed. On the basis of these results and based on the markedly increased number of lysosomes per ABCG2overexpressing MDR A549/K1.5 cell, we further explored the hypothesis that MDR cells may consequently become highly sensitive to the cytotoxic effect elicited by IAs upon lysosomal photodestruction, resulting in the overcoming of MDR. To this end, we first determined the cytotoxic activity of C-1330 in A549 and A549/K1.5 cells (Figures 3c and d, respectively) in the presence or absence of photosensitization. Illumination of C-1330-loaded cells resulted in a 6- and 14-fold decrease in the $I_{50}$ values in $A 549$ and A549/K1.5 cells, thereby resulting in an equal C-1330 sensitivity in both cell lines (Table 1).

We expanded these findings to other IAs; photosensitization markedly enhanced the cytotoxic activity of C-1375 and C-1379 in A549 and A549/K1.5 cells by 10-32-fold, relative to nonilluminated cells (Table 1 ). These results prompted us to further expand the scope of the cytotoxic impact of IAs upon photoexcitation to ABCB1 (P-gp)-overexpressing MDR nonsmall-cell lung cancer SW1573/2R160 cells and ABCC1 (MRP1)-overexpressing ovarian carcinoma 2008/MRP1 cells (Table 1). Photosensitization of C-1375-loaded MDR SW/2R160 and 2008/MRP1 cells resulted in a 13-51-fold decrease in the $\mathrm{IC}_{50}$ of $\mathrm{C}-1375$, whereas their parental cells were sensitized by factors of 24-36-fold. Remarkably, in the absence of illumination, these MDR cells displayed $I_{50}$ values in the range of 3.3-5.5 $\mu \mathrm{M}$, whereas photosensitization decreased the $\mathrm{IC}_{50}$ values to as low as $90-330 \mathrm{nM}$; we thus achieved a significant increase in parental cell sensitivity in three different types of MDR cancer cells.

To further strengthen the current finding that cell death occurs via lysosomal photodestruction we also evaluated the cytokskeletal structure of cells before or after lysosomal photodestruction by following F-actin using the well-established Texas Red-Phalloidin conjugate (Figures $3 e$ and $f$ ). Phalloidin is a bicyclic peptide belonging to a family of toxins isolated from the deadly Amanita phalloides and is widely used in fluorescence imaging applications to selectively label F-actin. Importantly, incubation with C-1330 followed by illumination led to lysosomal photodestruction, which resulted in highly damaged cells that were devoid of their cytoplasmic content as well as their actin cytoskeleton and only showed deformed residual cytoskeletal structure that was present in the cell's perimeter (Figure $3 \mathrm{f}$ ). In contrast, cells exposed to $\mathrm{C}-1330$ in the absence of illumination retained a normal actin cytoskeleton (Figure 3e). 

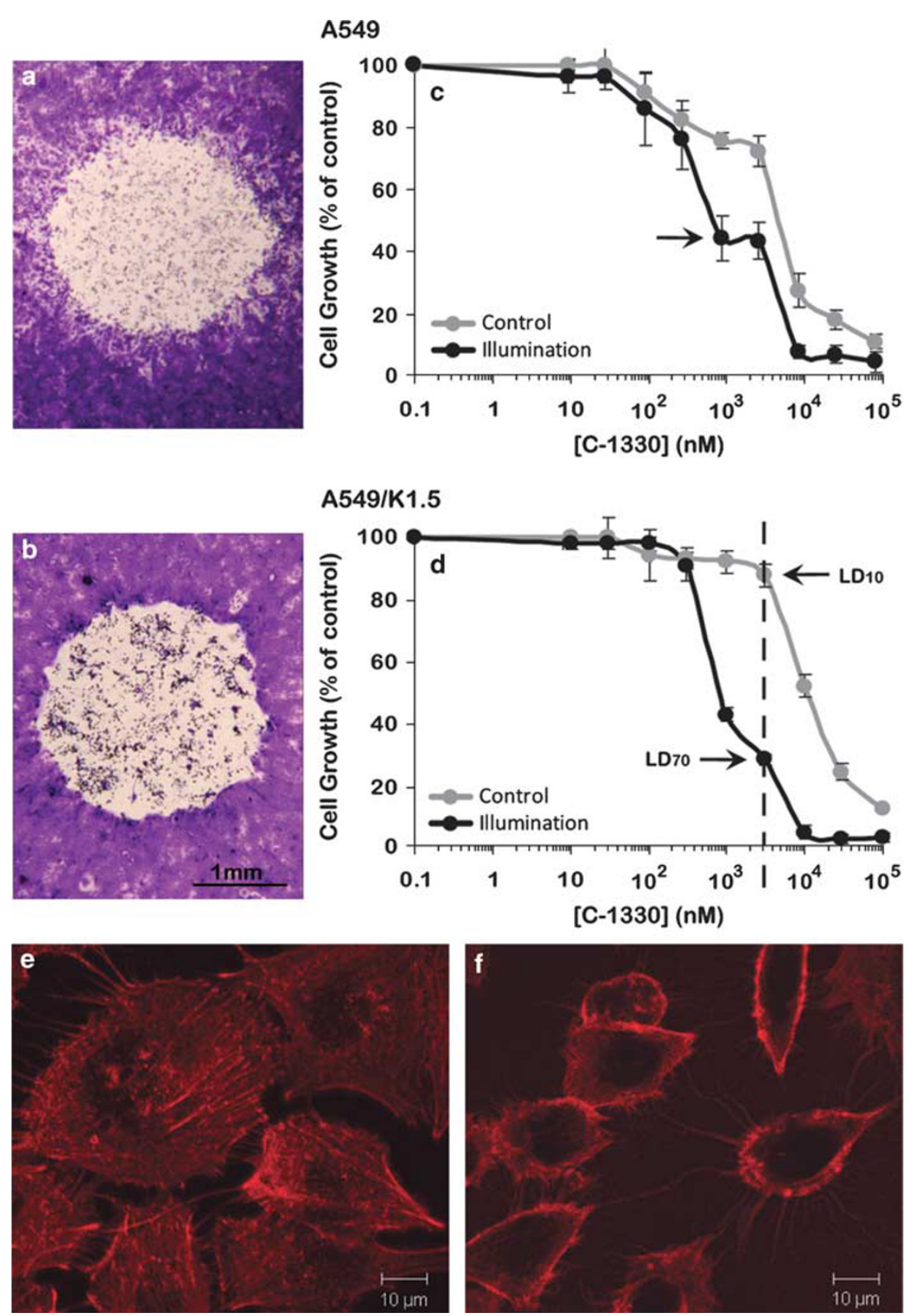

Figure 3 Photoexcitation of C-1330-loaded A549 and A549/K1.5 cells. Crystal Violet staining of C-1330-loaded cells was followed by exposure to confined blue light. A549 (a) and A549/K1.5 (b) cells were grown to half confluence and loaded with $10 \mu \mathrm{M} \mathrm{C}-1330$ for $1 \mathrm{~h}$. Under the light microscope, a confined zone of the cell monolayers was exposed for $1 \mathrm{~min}$ to a pulsed blue light beam $\left(\lambda_{\mathrm{ex}}=470 \mathrm{~nm}\right.$ with a measured fluence of $\left.\sim 180 \mathrm{~W} / \mathrm{m}^{2}\right)$. After $48 \mathrm{~h}$, monolayers were fixed with methanol, stained with Crystal Violet and analyzed by light microscopy at an $\times 8$ magnification. Cellular growth inhibition by C-1330 was determined as follows: logarithmically growing A549 cells (c) and their MDR A549/K1.5 subline (d) were exposed to various concentrations of C-1330 for $2 \mathrm{~h}$. To evaluate the effect of illumination on the cytotoxicity of C-1330, cells were divided into illuminated ( $1 \mathrm{~h}$ constant illumination which yielded a total fluence of $5 \mathrm{~J} / \mathrm{cm}^{2}$ ) and nonilluminated control cells. Cellular viability was determined using a Cell Proliferation Kit (XTT). Values depicted represent means \pm S.D. of at least three independent experiments. The F-actin cytoskeleton was evaluated with Texas Red-XPhalloidin in monolayer A549 cells; following 30 min incubation with $10 \mu \mathrm{M} \mathrm{C}-1330$ without illumination (e) or $2 \mathrm{~h}$ after photosensitzation (f), cells were incubated with Texas Red-X-Phalloidin and the cytoskeleton was analyzed by a fluorescence microscope as detailed in Materials and Methods. Note the highly damaged hollow cells that lost the cytoplasmic cytoskeletal structure due to lysosomal photodestruction after incubation with C-1330 and illumination, whereas cells incubated with C-1330 in the absence of illumination retained their intact cytoskeleton

\author{
Chloroquine induces a marked increase in both the \\ number and volume of lysosomes resulting in enhanced \\ C-1330 cytotoxicity upon photosensitization. Given the \\ remarkable cytotoxicity elicited by various IAs on tumor cells,
}

we explored the possibility of enhancing the number and volume of lysosomes in tumor cells. Previously it was shown that chloroquine, an anti-malarial and lysosomotropic agent, increased the number and volume of lysosomes in tumor 
Table 1 Growth inhibitory effects of various IAs on parental carcinoma cells and their MDR sublines

\begin{tabular}{|c|c|c|c|c|c|c|c|}
\hline \multirow[b]{2}{*}{ Drug } & \multirow[b]{2}{*}{ Cell line } & \multicolumn{2}{|c|}{ LD50 (nM) } & \multirow{2}{*}{$\begin{array}{c}\text { Fold } \\
\text { Sensitization }\end{array}$} & \multicolumn{3}{|c|}{ Survival at $3000 \mathrm{nM}^{\mathrm{a}}(\%)$} \\
\hline & & Control & Illumination & & Control & Illumination & Delta \\
\hline C-1330 & $\begin{array}{l}\text { A549 } \\
\text { A549/K1.5 }\end{array}$ & $\begin{array}{c}5023 \pm 716 \\
12269 \pm 1119\end{array}$ & $\begin{array}{l}842 \pm 208 \\
850 \pm 23\end{array}$ & $\begin{array}{c}6 \\
14\end{array}$ & $\begin{array}{l}72 \pm 5 \\
87 \pm 4\end{array}$ & $\begin{array}{l}43 \pm 6 \\
28 \pm 1\end{array}$ & $\begin{array}{l}29 \\
59\end{array}$ \\
\hline C-1379 & $\begin{array}{l}\text { A549 } \\
\text { A549/K1.5 }\end{array}$ & $\begin{array}{r}6349 \pm 867 \\
15192 \pm 580\end{array}$ & $\begin{array}{l}199 \pm 31 \\
747 \pm 45\end{array}$ & $\begin{array}{l}32 \\
20\end{array}$ & $\begin{array}{l}60 \pm 1 \\
82 \pm 4\end{array}$ & $\begin{array}{l}2 \pm 0.3 \\
7 \pm 0.1\end{array}$ & $\begin{array}{l}58 \\
75\end{array}$ \\
\hline C-1375 & $\begin{array}{l}\text { A549 } \\
\text { A549/K1.5 } \\
\text { SW1573 } \\
\text { SW1573/2R160 } \\
\text { 2008 } \\
\text { 2008/MRP1 }\end{array}$ & $\begin{array}{l}3054 \pm 260 \\
5264 \pm 877 \\
5535 \pm 596 \\
4384 \pm 125 \\
3280 \pm 345 \\
5115 \pm 889\end{array}$ & $\begin{aligned} 212 & \pm 29 \\
541 & \pm 56 \\
233 & \pm 5 \\
330 & \pm 56 \\
90 & \pm 9 \\
101 & \pm 0.1\end{aligned}$ & $\begin{array}{l}14 \\
10 \\
24 \\
13 \\
36 \\
51\end{array}$ & $\begin{array}{l}50 \pm 3 \\
70 \pm 5 \\
68 \pm 6 \\
74 \pm 3 \\
72 \pm 0.8 \\
80 \pm 2\end{array}$ & $\begin{array}{c}3 \pm 2 \\
3 \pm 0.6 \\
11 \pm 2 \\
24 \pm 4 \\
11 \pm 4 \\
13 \pm 3\end{array}$ & $\begin{array}{l}47 \\
67 \\
57 \\
50 \\
61 \\
67\end{array}$ \\
\hline
\end{tabular}

Abbreviations: IAs, imidazoacridinones; $\mathrm{LD}_{50}, 50 \%$ lethal dose; MDR, multidrug resistance

${ }^{a}$ Concentration chosen with the highest delta of $\%$ survival between illuminated and nonilluminated cells

cells. ${ }^{13}$ Fluorescence microscopy analysis after staining with LysoTracker red revealed that exposure of A549 cells to $20 \mu \mathrm{M}$ chloroquine for 24 (compare Figure 4c with $4 \mathrm{a}$ ) or $48 \mathrm{~h}$ (compare Figure $4 \mathrm{e}$ with $4 \mathrm{a}$ ) resulted in a marked dosedependent increase in the number and volume of lysosomes (Figure 4g); quantification using EZ-Quant software revealed an increase of 8.4- and 21.7-fold, respectively, in lysosomal fluorescence as compared with untreated control cells. We hence explored the impact of chloroquine pretreatment on C-1330 sensitivity in the presence and the absence of light exposure by growth inhibition assays. Consistent with the increased number and volume of lysosomes, chloroquine induced a marked time- and dose-dependent resistance of parental A549 cells to C-1330, in the absence of photosensitization; upon illumination, cells became 23- to 49fold more sensitive to C-1330, compared with the 6-fold photosensitization in the absence of chloroquine (Supplementary Table 1). These results indicate that chloroquine, currently used to treat malaria and rheumatoid arthritis, may be applied to increase the number and volume of lysosomes following which tumor cells may be efficiently eradicated by photosensitization of IA-loaded lysosomes.

In vivo antitumor activity of IAs using tumor xenografts. C-1375 and C-1379 showed similar spectroscopic characteristics with absorbance in the range 340-500 nm; their normalized fluorescence spectra are shown in Figure 5a. Both IAs emitted an intense fluorescence when excited at $444 \mathrm{~nm}$ with maximal emission at $553-554 \mathrm{~nm}$. Intravenous injection of these IAs into the chicken CAM model, followed by photosensitization produced a photodynamic vaso-occlusive activity for both compounds. When injected at a low dose ( $1 \mu \mathrm{g} / \mathrm{embryo}$; Figure $5 b$ ), the capillary network was massively occluded, whereas larger blood vessels remained perfused (see representative fluorescence angiographies taken $24 \mathrm{~h}$ postPDT in Figure 5c). Application of light alone did not induce a vaso-occlusive effect (data not shown). At a higher dose (100 $\mu \mathrm{g} / \mathrm{embryo})$, all blood vessels and capillaries were occluded within the illuminated area (Figure $5 \mathrm{~d}$ ). Interestingly, the uptake of IAs in lining endothelial cells was observed during and after PDT (Figure 5e), thereby also leading to vascular damage (Figure 5f). Furthermore, administration of IAs to the CAM grafted with human ovarian A2780 tumors followed by PDT, resulted in massive destruction of the tumor vasculature, already $24 \mathrm{~h}$ post-treatment (Figures $5 \mathrm{~g}$ and $\mathrm{h}$ ). A second PDT treatment was applied to the same tumors $24 \mathrm{~h}$ after the first treatment. As visualized $24 \mathrm{~h}$ after the repeated PDT, almost all tumor vasculature was destroyed and extensive vascular dysfunction was observed, as revealed by the profound leakage of FITC-dextran from the vasculature (Figures $5 \mathrm{i}$ and j). This was also accompanied by massive destruction of the ovarian tumor xenografts.

\section{Discussion}

Our current findings show that the achievement of an IAsdependent lysosomal photodestruction in tumor cells relies on the striking sequestration of IAs in lysosomes. Several lines of evidence support the differential compartmentalization of IAs within lysosomes of tumor cells: (i) parental A549 and their ABCG2-overexpressing MDR subline displayed co-localization of C-1330 and the established lysosomal marker, LysoTracker red; (ii) alkalinization of the acidic Iysosomal $\mathrm{pH}$ either with bafilomycin A1, a potent inhibitor of lysosomal $\mathrm{H}^{+}$-ATPase, or ammonium chloride, an established weak base $(\mathrm{pKa}=9.25)$ lysosomotropic alkalinization agent, resulted in the complete abrogation of lysosomal C-1330 accumulation. Previous studies have demonstrated that treatment with bafilomycin $\mathrm{A} 1$ and ammonium chloride increase the intralysosomal $\mathrm{pH}$ from $4-5$ to $6.5-7.0 .{ }^{14}$ Hence, the driving force for lysosomal sequestration of IAs is the acidic lysosomal $\mathrm{pH}$ and the consequent irreversible entrapment of IAs via protonation; and (iii) it is well documented ${ }^{15,16}$ that hydrophobic weak base chemotherapeutics, such as anthracyclines, Vinca alkaloids and other cytotoxic agents, including acridone-based fluorescent compounds, the latter of which are structurally related to IAs, markedly accumulate within acidic lysosomes; a hallmark example is acridine orange, a bona fide lysosomal marker widely used to fluorescently label lysosomes in viable cells. ${ }^{17}$ Thus, the organelle that predominantly compartmentalizes IAs in these cancer cells is the lysosome. However, one cannot exclude the possibility that alternative intracellular acidic vesicles such 

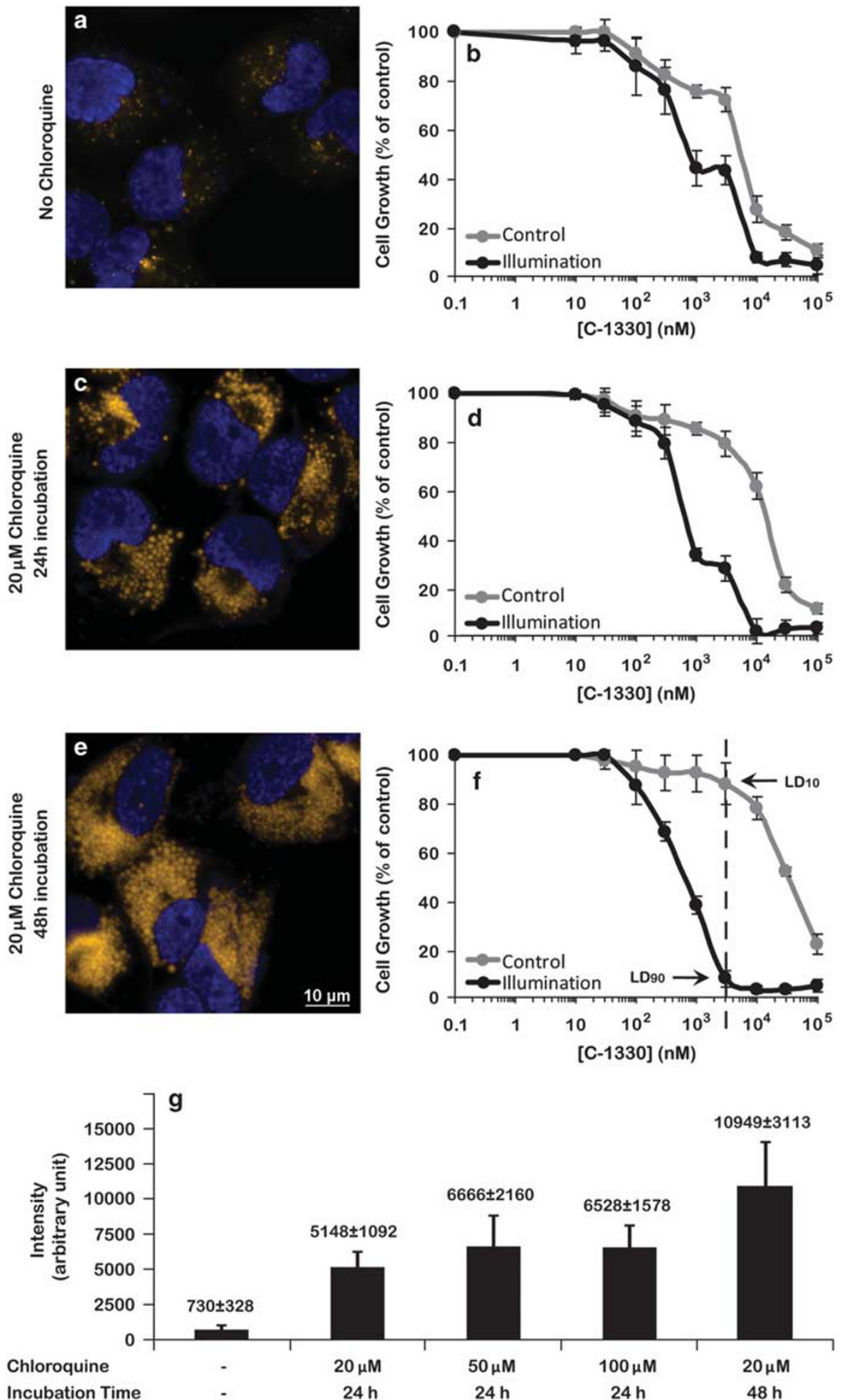

Figure 4 Treatment with the antimalarial chloroquine induces a marked increase in both the number and volume of lysosomes. A549 cells were seeded on glass cover plates, allowed to attach and then exposed to $20 \mu \mathrm{M}$ chloroquine for 24 (c) and $48 \mathrm{~h}(\mathbf{e})$, whereas control cells were not treated with chloroquine (a). The cytotoxic effect of $\mathrm{C}-1330$ to chloroquine-free control cells (b), cells exposed to $20 \mu \mathrm{M}$ chloroquine for 24 (d) or $48 \mathrm{~h}$ (f) in the absence or presence of illumination was determined by the XTT assay as described in Figure 3 legend. The dose- and time-dependent effect of incubation with chloroquine on the increased number of lysosomes per A549 cell as compared with nontreated cells was determined by fluorescence microscopy following staining with the viable lysosomal marker LysoTracker red (red fluorescence in chloroquine-treated cells) (g) as detailed in Materials and Methods. Note that for the sake of quantification accuracy, the red lysosomal fluorescence of LysoTracker was converted to a yellow fluorescence. Note the marked increase in the number and volume of lysosomes upon exposure of A549 cells to increasing concentrations of chloroquine as well as to prolonged incubation times. Live detection of nuclei was achieved by staining with $1 \mu \mathrm{g} / \mathrm{ml}$ Hoechst for $10 \mathrm{~min}$ before imaging 

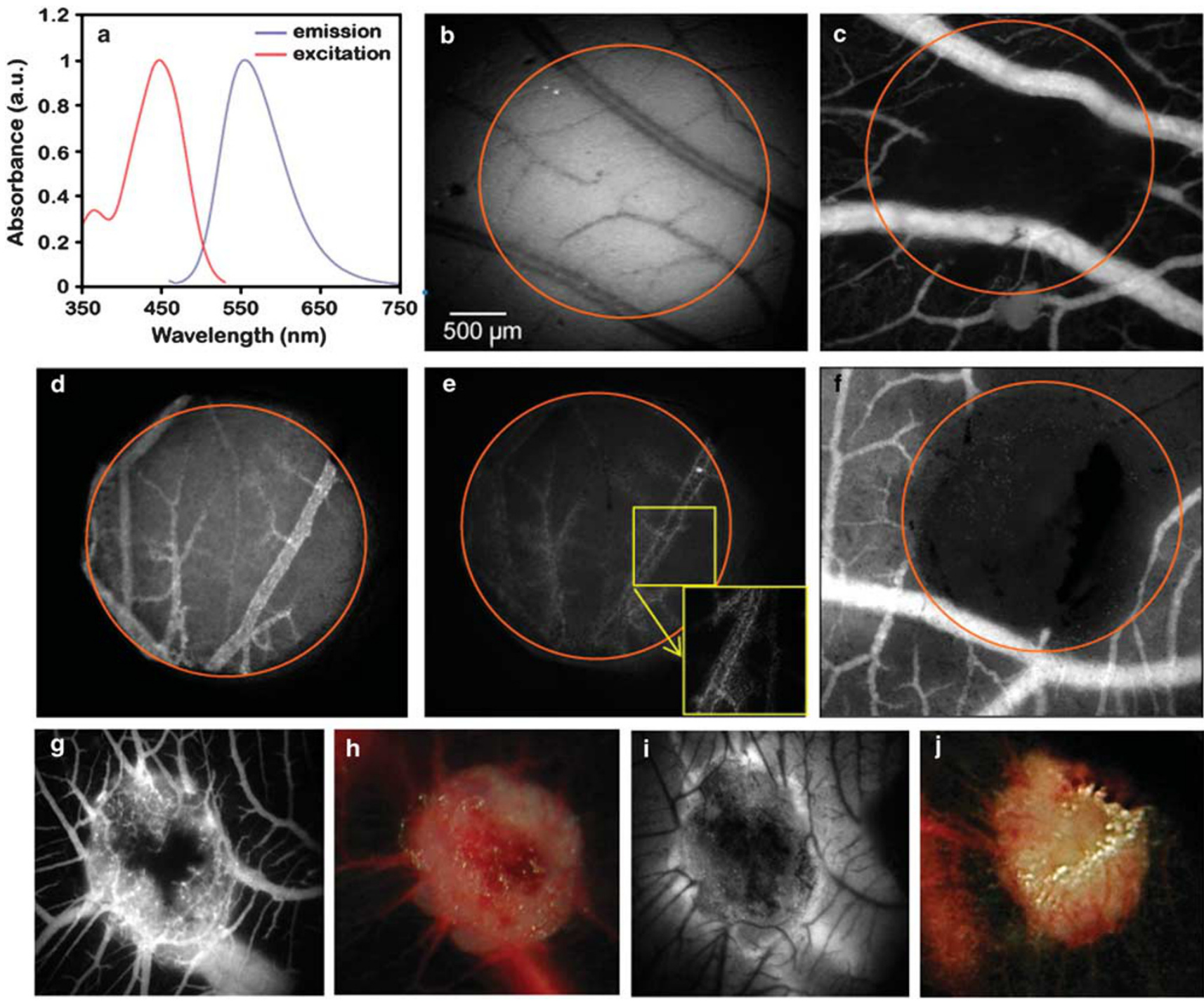

Figure 5 PDT after i.v. IA injection in the CAM vasculature and in tumor grafted CAMs. (a) Emission and excitation spectra of C-1379. (b) IA fluorescence angiography before IA-PDT $\left(1 \mu \mathrm{g} / \mathrm{embryo}, \lambda_{\mathrm{ex}}=420 \pm 20 \mathrm{~nm}\right)$. The bar in panel b denotes $500 \mu \mathrm{m}$. (c) FITC-dextran angiography $\left(\lambda_{\mathrm{ex}}=470 \pm 20 \mathrm{~nm}, \lambda_{\mathrm{em}}>520 \mathrm{~nm}\right), 24 \mathrm{~h}$ after PDT (light dose $20 \mathrm{~J} / \mathrm{cm}^{2}$, irradiance $\left.70 \mathrm{~mW} / \mathrm{cm}^{2}\right)$. (d) IA fluorescence angiography taken before IA-PDT $\left(100 \mu \mathrm{g} / \mathrm{embryo},\left(\lambda_{\mathrm{ex}}=420 \pm 20 \mathrm{~nm}\right)\right.$, right after (e; inset shows the magnification of IA in the endothelial lining after PDT) and $24 \mathrm{~h}(\mathrm{f})$ after PDT (light dose $20 \mathrm{~J} / \mathrm{cm}^{2}$, irradiance $270 \mathrm{~mW} / \mathrm{cm}^{2}$ ) visualized by FITC-dextran angiography $\left(\lambda_{\mathrm{ex}}=470 \pm 20 \mathrm{~nm}, \lambda_{\mathrm{em}}>520 \mathrm{~nm}\right)$. Tumor grafted CAMs, $24 \mathrm{~h}$ post IA-PDT of ovarian carcinoma on the CAM shown by fluorescence angiography $(\mathrm{g}$, FITC-dextran angiography, $\lambda_{\mathrm{ex}}=470 \pm 20 \mathrm{~nm}, \lambda_{\mathrm{em}}>520 \mathrm{~nm}$ ) and white light camera (h). The same tumor $48 \mathrm{~h}$ after the second IA-PDT visualized by FITC-dextran angiography (i) and white light camera (j). Black zones represent avascular areas

as recycling endosomes, trans-Golgi network and secretory vesicles could also contribute to sequestration of IAs. ${ }^{18}$

Our results suggest that an increased number of lysosomes is a key determinant of cell defense and consequent drug resistance against hydrophobic weak base cytotoxic agents including IAs that may reach their cellular target, topoisomerase II and DNA in the case of IAs. First, ABCG2-overexpressing $A 549 / K 1.5$ cells that displayed a marked increase in the number of lysosomes were 2.4-fold more resistant to C-1330 and C-1379, both of which are not recognized as drug efflux substrates by ABCG2. Second, the dramatic chloroquine dose-dependent induction of increased lysosome volume and number in parental A549 cells resulted in a consistent increase of 3-7-fold in C-1330-resistance. Hence, it is likely that epithelial and endothelial cells exposed to body fluids containing various toxicants have evolved to acquire larger number of lysosomes as means of cell defense against stress cues and cytotoxic agents including lipid-soluble weak base cytotoxins. ${ }^{19-21}$ Thus, as MDR cells are known to contain an increased number of lysosomes per cell, targeting MDR cells via lysosomal photodestruction using weak base photosenstizers may be readily seen as a pharmacological Achilles heel, and thus used as a Trojan horse approach to eradicate MDR tumor cells.

The following evidence supports the choice of targeting MDR cancer cells via IAs-dependent lysosomal photodestruction: (i) the luminal $\mathrm{pH}$ of lysosomes of various MDR tumor cells was found to be markedly more acidic than their parental counterparts, hence increasing the $\mathrm{pH}$ gradient between the cytoplasm and lysosomes; ${ }^{22,23}$ it is possible that the various 
MDR cell lines studied here also harbor more acidified lysosomes than their parental cells. This would result in an increased capacity of these MDR cells to sequester hydrophobic weak base cytotoxic agents including IAs within lysosomes; (ii) it has been shown that various MDR tumor cells display a more basic cytoplasmic $\mathrm{pH}$ than their drug-sensitive parental counterparts and nonmalignant cells. ${ }^{18,22,23}$ Consequently, the $\mathrm{pH}$ gradient between the cytoplasm and lysosomes will further increase, hence enhancing the capacity of lysosomes to concentrate hydrophobic weak base cytotoxic agents. Consistently, the MDR tumor cells employed here could comply with this increased $\Delta \mathrm{pH}$ between the lysosome and the cytoplasm, thereby further enhancing the capacity of lysosomes to highly sequester IAs. These predictions that the MDR cells we employed here possess lysosomes that are more acidic and presumably have a more basic cytoplasmic $\mathrm{pH}$ are supported by the fact that apart from the increased number of lysosomes in A549/ K1.5 cells, each lysosome of these MDR cells displayed a markedly increased accumulation of the fluorescent IAs, when compared with the rather faint fluorescence signal emitted from parental cell lysosomes; and (iii) one of the hallmarks of malignant tumors and metastatic cancers is acidosis of their tumor microenvironment; ${ }^{24}$ this is attributable to lactic acid production via anaerobic glycolysis of cancer cells (known as the Warburg phenomenon) that proliferate under hypoxic conditions. Hence, this acidosis of the tumor microenvironment implies that hydrophobic weak base chemotherapeutics would preferentially accumulate in solid tumors than in healthy tissues. This would present an excellent opportunity for intratumoral sequestration, which could be then followed by PDT, ROS generation and tumor destruction. This should result in enhanced therapeutic efficacy in the absence of severe damage to healthy tissues. In fact, this has been one of the major underlying principles of the selectivity of the concentration of the cytotoxic photosensitizer effect of PDT agents used against malignant cancers. $^{25}$

Our findings suggest that the mechanism underlying the damage inflicted by illumination, rapid lysosome rupture and cell death is mediated through rapid production of ROS. First, using established methodologies we showed that photoexcitation of IAs results in the formation of singlet oxygen and superoxide anion. Second, the presumed mediation of cell kill by ROS in our IA-loaded MDR cells undergoing lysosomal photodestruction, is in accord with the widely accepted mechanism of cytotoxicity of various photosensitizers used in PDT including protoporphyrins; upon illumination, these photosenstizers undergo fluorescence excitation (i.e., from the singlet excited state), and/or transferring their excitation (energy) to the triplet state molecular oxygen via intersystem crossing. The latter undergoes collisional energy transfer with ground state $\mathrm{O}_{2}^{3} \Sigma$ to yield reactive $\mathrm{O}_{2}^{1} \Delta$. Other ROS can be also produced by the excited photosensitizer. ${ }^{26}$ Recent studies have determined that ROS production in a PDT scheme using photoexcited protoporphyrin IX induced an essentially instantaneous production of ROS with a remarkable time rate constant of $0.0044 \mathrm{~min}^{-1} .^{27}$ Thus, the rapid lysosomal photodestruction in our MDR tumor cells is consistent with this rate constant of ROS production.
Singlet oxygen radicals and ROS are extremely detrimental to biomolecules such as proteins, nucleic acids and biomembranes and result in rapid cell death. Hence, the abrupt lysis of IA-loaded lysosomes upon photoexcitation is consistent with the rapid generation of ROS. The consequent intracellular rupture of lysosomes with the abrupt introduction of their harmful highly acidic content containing a spectrum of extremely potent hydrolytic enzymes would certainly lead to rapid digestion of polypeptides, nucleic acids and biomembranes and consequent cell lysis. This is supported by our Crystal Violet staining of monolayer cells undergoing lysosomal photodestruction in which complete cell lysis occurred with residual cytoskeletal scaffolds. In summary, the differential quantitative retention of the IAs photosensitizers in lysosomes in MDR cells could be used as an Achilles heel strategy to eradicate MDR cells. Interestingly, despite the photoexcitation, the harsh acidic $\mathrm{pH}$ and ample hydrolytic activities of the lysosomes, IAs, unlike many PDT agents, did not undergo photolysis and apparently retained their structure and function as they readily bind to DNA upon lysosomal rupture. This may be an added value in inflicting a cytotoxic effect upon neighbor nonilluminated malignant cells, tumor microenvironment and vasculature.

\section{Materials and Methods}

Chemicals. LysoTracker red DND99 and MitoTracker red CMXRos were purchased from Invitrogen (Carlsbad, CA, USA). Ammonium chloride, acridine orange, Crystal Violet and chloroquine were from Sigma-Aldrich (St. Louis, MO, USA). Bafilomycin A1 was purchased from Enzo Life Science (Farmingdale, NY, USA). IAs were synthesized by Drs. M Cholody, B Horowska and M Konieczny in the Department of Pharmaceutical Technology and Biochemistry, Gdansk University of Technology, Gdansk, Poland.

Cell culture. Non-small-cell lung cancer A549 cells and their ABCG2overexpressing A549/K1.5 subline, ovarian carcinoma 2008 cells and their stable MRP1 transfectant 2008/MRP1, non-small lung cancer cell line SW1573 and its ABCB1overexpressing subline SW1573/2R160 were maintained in RPMl-1640 medium (Gibco, Paisley, UK) supplemented with $10 \%$ fetal bovine serum, $2 \mathrm{mM}$ glutamine, $100 \mu \mathrm{g} / \mathrm{ml}$ penicillin and streptomycin (Biological Industries, Beth-Haemek, Israel) in a humid atmosphere containing $5 \% \mathrm{CO}_{2}$ at $37^{\circ} \mathrm{C}$. A549/K1.5 and SW1573/2R160 cells were grown in medium containing $1.5 \mu \mathrm{M} \mathrm{C}-1305{ }^{28}$ and $100 \mathrm{nM}$ doxorubicin, respectively. Cells were cultured in drug-free conditions for a week before any analysis.

Live cell imaging and quantification of lysosomes. A549 and A549/ $\mathrm{K} 1.5$ cells were grown for $24 \mathrm{~h}$ in $35 \mathrm{~mm}$ FluoroDish (World Precision Instruments, Sarasota, FL, USA). For C-1330 localization studies, cells were viably stained with $10 \mu \mathrm{M} \mathrm{C}-1330$ for $30 \mathrm{~min}$ along with either $100 \mathrm{nM}$ LysoTracker red or $100 \mathrm{nM}$ MitoTracker red for $1 \mathrm{~h}$ at $37^{\circ} \mathrm{C}$. The established viable lysosomal marker LysoTracker red was used to quantify the number/volume of lysosomes per cell in parental tumor cells and their MDR sublines overexpressing specific MDR efflux transporters of the ABC superfamily. Cellular LysoTracker red fluorescence was followed with a Zeiss Axiovert 200 inverted fluorescence microscope (Carl Zeiss, Oberkochen, Germany) equipped with a digital camera using a red Cye 3 filter $\left(\lambda_{\mathrm{ex}}=545 \mathrm{~nm}\right.$ and $\lambda_{\mathrm{em}}=605 \mathrm{~nm}$ ). Quantification of red fluorescence of lysosomes per cell was undertaken by evaluating at least 25 LysoTracker red-stained cells in each of at least three independent experiments using the EZ-Quant statistical analysis software (version 1105). Inverted fluorescence microscopy analysis of viable fluorescent cells was performed at a magnification of $\times 630$.

Before fluorescence imaging, cells were incubated in medium containing $2 \mu \mathrm{g} / \mathrm{ml}$ Hoechst 33342 for $10 \mathrm{~min}$. For nuclear staining, A549/K1.5 cells were incubated for $1 \mathrm{~h}$ with Hoechst 33342 and $10 \mu \mathrm{M}$ fumitremorgin $\mathrm{C}(\mathrm{FTC})$ to prevent efflux of the DNA dye by ABCG2. Live cell imaging was performed using a Zeiss Axiovert 200 inverted fluorescent microscope (Carl Zeiss).

Chloroquine treatment. A549 cells were seeded in 96-well plates at 500 or 1000 cells/well (for $24 \mathrm{~h}$ or $48 \mathrm{~h}$ treatment, respectively; $0.1 \mathrm{ml}$ medium $/$ well). 
Following an overnight incubation, chloroquine was added for $24 \mathrm{~h}(20,50$ and $100 \mu \mathrm{M})$ or $48 \mathrm{~h}(20 \mu \mathrm{M})$. For cytotoxicity assays, C-1330 concentrations ranging from $10 \mathrm{nM}$ to $100 \mu \mathrm{M}$ were added for two additional hours. For viable fluorescent labeling of lysosomes and nuclei, LysoTracker red DND $99(100 \mathrm{nM})$ was added for $1 \mathrm{~h}$ before fluorescence imaging, following which Hoechst $33342(2 \mu \mathrm{g} / \mathrm{ml})$ was added $10 \mathrm{~min}$ before imaging. Cells were first analyzed with a Zeiss Axiovert 200 inverted fluorescence microscope (Carl Zeiss) equipped with a digital camera and then illuminated using a GFP + DsRed filter $\left(\lambda_{\text {ex }}=470 \mathrm{~nm} ; \lambda_{\text {em }}=512 \mathrm{~nm}\right.$ and $630 \mathrm{~nm}$; this is a long pass filter which transmits longer wavelengths over a wide spectrum). LysoTracker Red and MitoTracker Red CMXRos were followed using a red filter Cye $3\left(\lambda_{\mathrm{ex}}=545 \mathrm{~nm}\right.$ and $\left.\lambda_{\mathrm{em}}=605 \mathrm{~nm}\right)$, whereas a DAPI filter $\left(\lambda_{\mathrm{ex}}=365 \mathrm{~nm}\right.$ and $\lambda_{\mathrm{em}}=445 \pm 50 \mathrm{~nm}$ ) was used to follow nuclear staining with Hoechst 33342 . Light intensity was maintained at $12.5 \%$ of the maximum. Light intensity in the illuminated cell monolayer zone was $\sim 3 \mathrm{~W} / \mathrm{m}^{2}$ and the light dosage for $30 \mathrm{~s}$ was $90 \mathrm{~J} / \mathrm{m}^{2}$. The magnification used was $\times 630$.

Crystal Violet staining. Cells were grown to confluence on glass bottom plates and incubated with $10 \mu \mathrm{M} \mathrm{C}-1330$ for $30 \mathrm{~min}$. Following medium refreshment, plates were exposed to $1 \mathrm{~min}$ constant excitation at $470 \mathrm{~nm}$ using a Zeiss inverted fluorescence microscope. Cells displaying lysosomal photodestruction were further incubated for $48 \mathrm{~h}$ at $37^{\circ} \mathrm{C}$, then washed twice with PBS, fixed with $70 \%$ and stained with a dilute (1:5) Crystal Violet solution in PBS. Stained monolayer cells were then examined and photographed using a Leica M80 binocular at a $\times 8$ magnification (Leica Microsystems GmbH, Wetzlar, Germany).

Cytotoxicity assays. Cells were seeded in 96-well plates $\left(2 \times 10^{4}\right.$ cells in $90 \mu \mathrm{l}$ growth medium/well), incubated for $24 \mathrm{~h}$ and exposed to increasing concentrations of IAs. Following $2 \mathrm{~h}$ incubation, the drug-containing medium was replaced by drug-free medium and cells were placed on an illuminator plate (Model 460 thin line series, S\&S X-Ray Products Inc., Brooklyn, NY, USA) for $1 \mathrm{~h}$, hence achieving a light dose of $5 \mathrm{~J} / \mathrm{cm}^{2}$. Plates were then further incubated for $24 \mathrm{~h}$. Cell viability was determined using the Cell Proliferation Kit (XTT) (Biological Industries). Drug concentrations required to inhibit cell growth by $50 \%\left(\mathrm{IC}_{50}\right)$ in the absence or presence of illumination, were determined from the cell survival curves. Results presented are means of at least three independent experiments.

Spectroscopy assessment of IAs. C-1375 and C-1379 were dissolved in DMSO (Sigma-Aldrich) and diluted in $0.9 \% \mathrm{NaCl}$. Absorption spectra were recorded with a two-beam Varian Cary UV-Vis-NIR 500 Scan spectrophotometer in 1-cm long quartz cuvettes (Suprasil, Hellma, Müllheim, Germany) in the range of 350 to $750 \mathrm{~nm}$ with an average scan speed of $600 \mathrm{~nm} / \mathrm{min}$ at room temperature. Steadystate luminescence spectra were recorded with a luminescence spectrometer (Perkin-Elmer, model LS50B, Wellesley, MA, USA).

Texas red-phalloidin staining. A549 cells were grown on coverslips in 24-well plates until confluence was reached. Cells were then incubated with $10 \mu \mathrm{M}$ C-1330 for $30 \mathrm{~min}$ and exposed to light for $1 \mathrm{~min}$, resulting in lysosoma photodestruction. Control cells were loaded with $\mathrm{C}-1330$ but were not illuminated. C1330-loaded cells were either fixed and stained with Texas Red-X-Phalloidin (Life Technologies, Carlsbad, CA, USA) with no illumination or stained with Texas Red-XPhalloidin $2 \mathrm{~h}$ after light-induced lysosomal photodestruction. Cells were then washed twice with PBS and fixed by adding $4 \%$ formaldehyde for $10 \mathrm{~min}$ in room temperature. Following two additional washes with PBS, $0.1 \%$ Triton $X-100$ was added for $10 \mathrm{~min}$ and then washed twice to remove Triton X-100. Blocking was then achieved using a solution of $20 \%$ skim milk and $2.5 \%$ fetal calf serum for $20 \mathrm{~min}$. At this stage, $5 \mu$ l of Texas Red-X phalloidin stock solution was added into $0.2 \mathrm{ml}$ of PBS and incubated protected from light for $20 \mathrm{~min}$. The samples were finally rinsed to remove excess Texas Red-X Phalloidin. Coverslips were removed gently from wells and attached onto slides using Fluoromount-G. Fluorescent cells were analyzed by a fluorescence microscope at $\lambda_{\mathrm{ex}}=545 \mathrm{~nm}$ and $\lambda_{\mathrm{em}}=605 \mathrm{~nm}$.

Detection of superoxide anion and singlet oxygen. Photosensitized formation of superoxide anion was detected by EPR-spin trapping using DMPO as a spin trap, as described previously. ${ }^{29,30}$ IAs solutions containing $0.1 \mathrm{M}$ DMPO (Sigma-Aldrich Chemie GmbH, Steinheim, Germany) in DMSO/ $\mathrm{H}_{2} \mathrm{O}(9: 1)$ were illuminated in a 0.25 -mm quartz cell within the resonant cavity of a Bruker EMEX-AA EPR spectrometer (Bruker BioSpin, Rheinstetten, Germany) with 400-490 nm light derived from a Cermax PE300CE-13FM $300 \mathrm{~W}$ lamp in air-cooled housing (Perkin Elmer). This light source was equipped with the necessary combination of cutoff and band-pass filters. Relative yields of the photo-formation of superoxide anion by IAs in the presence or absence of NADH, used as an auxiliary electron donor, were determined by comparing rates of the accumulation of DMPO-OOH determined for IAs and for riboflavin with and without EDTA. Quantum yields for singlet oxygen photogeneration by IAs were determined relative to that for Rose Bengal (RB) by measuring time-resolved luminescence intensity in DMSO or acetonitrile solutions at $1270 \mathrm{~nm}$, after excitation of the compounds or RB at $355 \mathrm{~nm}$ with microjoule $750 \mathrm{ps}$ pulses generated by a microchip Nd:YAG laser (Pulselas-P-1064-FC, Alphalas GmbH, Goettingen, Germany), operating with $2-10 \mathrm{kHz}$ repetition rate. Near-infrared luminescence was measured perpendicularly to the excitation beam in a photoncounting mode ${ }^{31}$ using a thermoelectric cooled NIR PMT module (model H10330-45, Hamamatsu, Shizuoka, Japan) equipped with a 1100-nm cutoff filter and additional selected narrow-band filters (NB series, NDC Infrared Engineering Ltd, Maldon, UK). Data were collected using a computer-mounted $\mathrm{PCl}$-board multichannel scaler (NanoHarp 250, PicoQuant GmbH, Berlin, Germany).

In vivo PDT with IAs using the CAM model. Fertilized chicken eggs (Animalco AG, Staufen, Switzerland) were incubated in a hatching incubator $(65 \%$ humidity, $37^{\circ} \mathrm{C}$ ) equipped with an automatic rotator (Savimat, Chauffry, France) as described previously. ${ }^{32}$ Briefly, on embryo development day 11 (EDD 11), the hole in the shell above the air pouch of the egg (made on EDD 3) was extended to a diameter of $\sim 3 \mathrm{~cm}$ in order to provide better access to the CAM for the experiments. Eggs were placed under an epi-fluorescence microscope (Eclipse 600 FN, Nikon, Egg, Zurich, Switzerland). Subsequently, $100 \mu \mathrm{l}$ of IAs (C-1375 or $\mathrm{C}$-1379) at doses of $1 \mu \mathrm{g} / \mathrm{embryo}$ or $100 \mu \mathrm{g} / \mathrm{embryo}$ were administered into the main vessel of the CAM through a 33-gauge needle fitted to a $100 \mu \mathrm{l}$ syringe (Hamilton, Reno, NV, USA). One minute later, the $0.02 \mathrm{~cm}^{2}$ area of the CAM was irradiated with a light dose of $20 \mathrm{~J} / \mathrm{cm}^{2}\left(\lambda_{\mathrm{ex}}=420 \mathrm{~nm}\right)$, irradiance of 70 or $270 \mathrm{~mW} / \mathrm{cm}^{2}$. The site was photographed before and after the irradiation. Subsequently, the egg opening was sealed with Parafilm (Pechiney, Menasha, WI, USA) and the embryo was incubated for $24 \mathrm{~h}$. The PDT-induced damage was assessed by fluorescence angiography after i.v. injection of FITC-dextran $\left(20 \mathrm{kDa}, 25 \mathrm{mg} / \mathrm{ml} ; \lambda_{\mathrm{ex}}=470 \mathrm{~nm}\right.$ and $\left.\lambda_{\mathrm{em}}=520 \mathrm{~nm}\right)$ and India ink (30 $\mu$ l/embryo) into the extra-embryonic cavity as previously described. ${ }^{33}$ IA treatment was performed with five eggs.

Tumor xenografts on the CAM and IA treatment with PDT. Human A2780 ovarian carcinoma cells were used to establish tumor xenografts in the CAM. Tumor cells were suspended in medium containing 20\% methocel (Sigma-Aldrich), and $50 \mu \mathrm{l}$ drops $\left(1 \times 10^{6} \mathrm{cells} / \mathrm{drop}\right)$ were dispensed on the internal part of the lid of a petri dish. After $24 \mathrm{~h}$, spheroids were harvested and placed on the CAM (EDD 7). At EDD $11, \mathrm{C}-1375$ and C-1379 were injected i.v. at a dose of $12 \mu \mathrm{g} / \mathrm{embryo}$. PDT of tumor xenografts was performed 1 min later $\left(\lambda_{\mathrm{ex}}=420 \mathrm{~nm}\right.$; light dose $34 \mathrm{~J} / \mathrm{cm}^{2}$; irradiance $\left.70 \mathrm{~mW} / \mathrm{cm}^{2}\right)$. The PDT procedure was repeated $24 \mathrm{~h}$ later. Tumors were visualized by standard white light camera and subsequently by FITC-dextran angiography, 24 and $72 \mathrm{~h}$ after the first PDT treatment.

\section{Conflict of Interest}

The authors declare no conflict of interest.

1. Skladanowski A, Plisov SY, Konopa J, Larsen AK. Inhibition of DNA topoisomerase II by imidazoacridinones, new antineoplastic agents with strong activity against solid tumors. Mol Pharmacol 1996; 49: 772-780.

2. Kusnierczyk H, Cholody WM, Paradziej-Lukowicz J, Radzikowski C, Konopa J. Experimental antitumor activity and toxicity of the selected triazolo- and imidazoacridinones. Arch Immunol Ther Exp (Warsz) 1994; 42: 415-423.

3. Dziegielewski J, Slusarski B, Konitz A, Skladanowski A, Konopa J. Intercalation of imidazoacridinones to DNA and its relevance to cytotoxic and antitumor activity. Biochem Pharmacol 2002; 63: 1653-1662.

4. Borst P, Elferink RO. Mammalian ABC transporters in health and disease. Annu Rev Biochem 2002; 71: 537-592.

5. Haimeur A, Conseil G, Deeley RG, Cole SP. The MRP-related and BCRP/ABCG2 multidrug resistance proteins: biology, substrate specificity and regulation. Curr Drug Metab 2004; 5: 21-53.

6. Sarkadi B, Ozvegy-Laczka C, Nemet K, Varadi A. ABCG2 - a transporter for all seasons. FEBS Lett 2004; 567: 116-120.

7. Gottesman MM, Fojo T, Bates SE. Multidrug resistance in cancer: role of ATP-dependent transporters. Nat Rev Cancer 2002; 2: 48-58.

8. Szakacs G, Paterson JK, Ludwig JA, Booth-Genthe C, Gottesman MM. Targeting multidrug resistance in cancer. Nat Rev Drug Discov 2006; 5: 219-234. 
9. Assaraf YG. Molecular basis of antifolate resistance. Cancer Metastasis Rev 2007; 26: 153-181.

10. Lim SH, Nowak-Sliwinska P, Kamarulzaman FA, van den Bergh H, Wagnieres G, Lee HB The neovessel occlusion efficacy of 15-hydroxypurpurin-7-lactone dimethyl ester induced with photodynamic therapy. Photochem Photobiol 2010; 86: 397-402.

11. Bram EE, Adar Y, Mesika N, Sabisz M, Skladanowski A, Assaraf YG. Structural determinants of imidazoacridinones facilitating antitumor activity are crucial for substrate recognition by ABCG2. Mol Pharmacol 2009; 75: 1149-1159.

12. Korinek M, Dedic R, Svoboda A, Hala J. Luminescence study of singlet oxygen production by meso-tetraphenylporphine. J Fluoresc 2004; 14: 71-74.

13. Fan $C$, Wang W, Zhao B, Zhang S, Miao J. Chloroquine inhibits cell growth and induces cell death in A549 lung cancer cells. Bioorg Med Chem 2006; 14: 3218-3222.

14. Christensen KA, Myers JT, Swanson JA. pH-dependent regulation of lysosomal calcium in macrophages. J Cell Sci 2002; 115 (Pt 3): 599-607.

15. Beijnena JH, Bultb A, Underberg WJM. Mitoxantrone Hydrochloride. Anal Profiles Drug Subs 1988; 17: 221-258.

16. Burns JH. Vincristine sulfate. Anal Profiles Drug Subs 1972; 1: 463-480.

17. Agostinelli E, Condello M, Molinari A, Tempera G, Viceconte N, Arancia G. Cytotoxicity of spermine oxidation products to multidrug resistant melanoma M14 ADR2 cells: sensitization by the MDL 72527 lysosomotropic compound. Int J Oncol 2009; 35: 485-498.

18. Altan N, Chen Y, Schindler M, Simon SM. Defective acidification in human breast tumor cells and implications for chemotherapy. J Exp Med 1998; 187: 1583-1598.

19. Groth-Pedersen L, Ostenfeld MS, Hoyer-Hansen M, Nylandsted J, Jaattela M. Vincristine induces dramatic lysosomal changes and sensitizes cancer cells to lysosome-destabilizing siramesine. Cancer Res 2007; 67: 2217-2225.

20. Jansen G, Barr H, Kathmann I, Bunni MA, Priest DG, Noordhuis $P$ et al. Multiple mechanisms of resistance to polyglutamatable and lipophilic antifolates in mammalian cells: role of increased folylpolyglutamylation, expanded folate pools, and intralysosomal drug sequestration. Mol Pharmacol 1999; 55: 761-769.

21. Trifillis AL, Hall-Craggs M. Effects of cyclosporine on human endothelial cell cultures. Toxicol Pathol 1986; 14: 210-212.

22. Schindler M, Grabski S, Hoff E, Simon SM. Defective $\mathrm{pH}$ regulation of acidic compartments in human breast cancer cells (MCF-7) is normalized in adriamycin-resistant cells (MCF-7adr). Biochemistry 1996; 35: 2811-2817.

23. Larsen AK, Escargueil AE, Skladanowski A. Resistance mechanisms associated with altered intracellular distribution of anticancer agents. Pharmacol Ther 2000; 85: 217-229.
24. Gillies RJ, Gatenby RA. Hypoxia and adaptive landscapes in the evolution of carcinogenesis. Cancer Metastasis Rev 2007; 26: 311-317.

25. Moore CM, Pendse D, Emberton M. Photodynamic therapy for prostate cancer-a review of current status and future promise. Nat Clin Pract Urol 2009; 6: 18-30.

26. van den Bergh H, Ballini JP. Photodynamic therapy: basic principle. In: Frankhauser F, Kwasniewska S (eds). Lasers in Ophthalmology: Basic, Diagnostic and Surgical Aspects. Kugler Publications: The Hague, The Netherlands, 2003, pp 183-195.

27. Radu IA, Moisescu M, lancu D, Savopol T, Kovacs E. Ala uptake and laser induced ros production in keratinocytes. Romanian J Biophysics 2008; 18: 265-272.

28. Bram EE, Ifergan I, Grimberg M, Lemke K, Skladanowski A, Assaraf YG. C421 allele-specific ABCG2 gene amplification confers resistance to the antitumor triazoloacridone C-1305 in human lung cancer cells. Biochem Pharmacol 2007; 74 41-53.

29. Panzella L, Szewczyk G, d'Ischia M, Napolitano A, Sarna T. Zinc-induced structural effects enhance oxygen consumption and superoxide generation in synthetic pheomelanins on UVA/visible light irradiation. Photochem Photobiol 2010; 86: 757-764.

30. Zareba M, Szewczyk G, Sarna T, Hong L, Simon JD, Henry MM et al. Effects of photodegradation on the physical and antioxidant properties of melanosomes isolated from retinal pigment epithelium. Photochem Photobiol 2006; 82: 1024-1029.

31. Jimenez-Banzo A, Ragas X, Kapusta P, Nonell S. Time-resolved methods in biophysics. 7 . Photon counting versus analog time-resolved singlet oxygen phosphorescence detection. Photochem Photobiol Sci 2008; 7: 1003-1010.

32. Nowak-Sliwinska $P$, van Beijnum JR, van Berkel $M$, van den Bergh $H$, Griffioen AW. Vascular regrowth following photodynamic therapy in the chicken embryo chorioallantoic membrane. Angiogenesis 2010; 13: 281-292.

33. Nowak-Sliwinska P, Ballini JP, Wagnieres $G$, van den Bergh $H$. Processing of fluorescence angiograms for the quantification of vascular effects induced by anti-angiogenic agents in the CAM model. Microvasc Res 2010; 79: 21-28.

(c)

Cell Death and Disease is an open-access journal published by Nature Publishing Group. This work is licensed under the Creative Commons Attribution-Noncommercial-No Derivative Works 3.0 Unported License. To view a copy of this license, visit http://creativecommons.org/licenses/by-nc-nd/3.0/

\section{Supplementary Information accompanies the paper on Cell Death and Disease website (http://www.nature.com/cddis)}

